\title{
Reaction of dioxygen with a cross-conjugated carbon-carbon double bond in a bis-macrocycle diiron compound
}

\author{
D. Brent MacQueen ${ }^{\text {a }}$, Christopher Lange a , Melvin Calvin a , John W. Otvos a, \\ Larry O. Spreer ${ }^{\mathrm{b}}$, Christian B. Allan ${ }^{\mathrm{b}}$, Anna Ganse ${ }^{\mathrm{b}}$, Richard B. Frankel ${ }^{\mathrm{c}}$ \\ a Deparment of Chemistry and Lawrence Berkeley National Laboratory. University of California. Berkeley. CA 94720, USA \\ 'Department of Chemistry, University of the Pacific, Stockton. CA 95211. USA \\ - Deparment of Physics, Califormia Polytechnic State University. San Luis Opispo. CA 93407. USA
}

\begin{abstract}
Dioxygen at atmospheric pressure attacks a cross-conjugated carbon-carbon double bond in a diiron complex to form two, like, keto macrocyclic iron(I1) complexes. This reaction occurs with high yield in both solution and in the solid state. A dioxetane intermediate is, therefore, invoked. The rate of the reaction is very cependent on the nature of the axial ligands on the low-spin iron(II) ions in the bimetallic complex. The rate is at least a factor of $10^{4}$ faster with DMF ligands than with $\mathrm{CH}_{3} \mathrm{CN}$ axial ligands. This rate dependence is explained by stabilization of a peroxo biradical transition state en route to a dioxetane intermediate. The keto-macrocyle product has the carbonyl group conjugated with a $\beta$-diimine in a six-membered chelate ring. The conformation of this keto macrocycle is fixed on the NMR time scale and the spectra of all ten non-equivalent protons in the complex can be unambiguously assigned. The keto $\beta$-dimine ligand is an excellent $\pi$-acceptor as indicated by the high $\mathrm{Fe}$ (II) to Fe(III) oxidation potential of the compound and by the Mössbauer spectrum, which shows a low value for the center shift and a high value for the quadrupole splitting parameter.
\end{abstract}

Keywords: Iron complexes; Macrocyclic ligand complexes; Dioxygen complexes

\section{Introduction}

There are many different types of reactions of molecular oxygen with transition metal complexes. These include removal of electrons from the metal center or from the coordinated ligand, oxidative dehydrogenation of the ligand and formation of metal-oxygen adducts. This paper describes a novel reaction in which a cross-conjugated carbon-carbon double bond in a diiron complex, 1, reacts with dioxygen to break the double bond and form two keto-macrocyclic iron complexes, 2 , shown below. This reaction occurs in inigh yield with atmospheric pressure dioxyger. at room temperature in the dark.

We recently reported [1] the structure and properties of a new type of bimetallic complex that contains a cross-conjugated $\beta$-diimine linkage between metals in two different macrocycles, 1 . One of our first observations of the diiron complex with axial acetonitrile ligands was that solid samples of the perchlorate salt underwent a slow reaction if exposed to the atmosphere. The solubility of $\mathbf{2}$ is much greater than that of 1 and the surface film can be removed by rapid washing with acetonitrile. In acetonitrile solution, the blue product has a $\lambda_{\max }$ at $650 \mathrm{~nm}$. The same blue product slowly formed in aerated acetonitrile solution. We have isolated and identified the blue species as the monomeric $\beta$-diimine keto macrocycle, 2, with axial acetonitrile ligands. A much faster reaction occurs in aerated DMF to produce an olive-green $\left(\lambda_{\max }=720\right.$ $\mathrm{nm}) \beta$-diimine keto-macrocycle with DMF axial ligands.

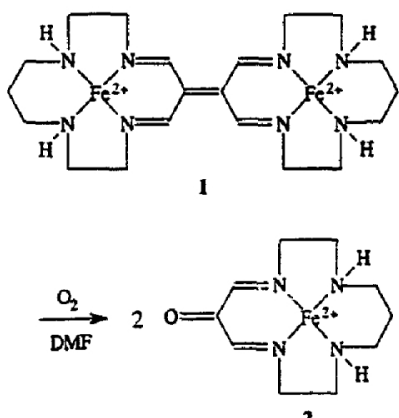


The attack of molecular oxygen on the bridging carboncarbon double bond produces a very high percentage yield ( $>95 \%$ ) of the keto-macrocycie product. The high yield and the fact that the reaction also occurs in the solid state suggests that a dioxetane intermediate is involved.<smiles>CC1(C)OOC1(C)C</smiles>

A mechanism for the formation of the dioxetane intermediate, which involves oxidation of one of the iron atoms, is proposed to explain the apparent absence of similar examples in organic systems and the very large axial ligand dependence of the rate of formation.

The proton NMR spectrum of the keto species was critical in its identification and is particularly interesting in that the spectra of ail ten distinct protons of the macrocycle can be unambiguously assigned. The Mössbauer spectrum of the compound is also reported and is compared to other low-spin iron-II tetraaza macrocycles. The solvent dependence of the optical spectrum, the electrochemical behavior and the rate of formation are discussed.

\section{Experimental}

\subsection{Materials and methods}

All solvents used were reagent grade $(99+\%)$ and dried over activated molecular sieves ( $3 \AA$ ) except acetonitrile which was purchased from Burdick and Jackson and used directly. In the electrochemical measurements $\mathrm{CH}_{3} \mathrm{CN}$ was distilled from calcium hydride prior to use. All reagents were the highest quality commercially available and used without purification. Instruments used were as follows: FT-IR, Nicolet 5DX; NMR, Varian Gemini 300 broadband system; UV-VIS-NIR, Perkin-Elmer Array 3840 with 7500 professional computer or a Perkin-Elmer Lambda 9. Cyclic voltammograms were measured under argon with an IBM EC $225^{2 \mathrm{~A}}$ or a PAR 175/173 system with tetrabutylammonium hexafluorophosphate as the electrolyte. Conditions: Pt disk working electrode; $0.1 \mathrm{M}$ electrolyte; scan rate $200 \mathrm{mV} \mathrm{s}^{-1}$. Electrospray mass spectra were measured in a $50 \%$ aqueous acetonitrile solution (UC Berkeley Mass Spec Labs). Mössbauer spectra were obtained by Richard Frankel.

\section{I.J. $\left[\mathrm{Fe}_{2}\left(\mathrm{C}_{20} \mathrm{H}_{34} \mathrm{~N}_{8}\right)\left(\mathrm{CH}_{3} \mathrm{CN}\right)_{2}\right]\left(\mathrm{ClO}_{4}\right)_{4}(\mathrm{I})$}

$\left[\mathrm{Fe}_{2}\left(\mathrm{C}_{20} \mathrm{H}_{34} \mathrm{~N}_{8}\right)\left(\mathrm{CH}_{3} \mathrm{CN}\right)_{2}\right]\left(\mathrm{ClO}_{4}\right)_{4}$ was prepared by acrobic oxidative dehydrogenation of $\left[\mathrm{Fe}\left(\mathrm{C}_{10} \mathrm{H}_{24} \mathrm{~N}_{4}\right)\left(\mathrm{CH}_{3}\right.\right.$ $\left.\mathrm{CN})_{4}\right]\left(\mathrm{ClO}_{4}\right)_{3}$ as previously reported [2]. Caution: perchlorate salts are potentially explosive. Avoid scraping compounds with metal spatulas and do not filter with sintered glass filters.
2.1.2. Preparation of $\left[\mathrm{Fe}\left(\mathrm{C}_{10} \mathrm{H}_{18} \mathrm{~N}_{4} \mathrm{O}\right)(\mathrm{DMF})_{2}\right](\mathrm{ClO})_{4}$

A solution of complex $1(0.50 \mathrm{~g}, 0.47 \mathrm{mmol})$ in DMF (25 ml) was stirred for about $1 \mathrm{~h}$ open to air. After the initial brown color turned dark green a four-fold excess of diethyl ether was added. The solution was filtered and the powdery green solid washed with diethyl ether. Yield: $0.490 \mathrm{~g}(0.80$ mmol, $85 \%$ ).

\subsubsection{Preparation of $\left[\mathrm{Fe}\left(\mathrm{C}_{10} \mathrm{H}_{18} \mathrm{~N}_{4} \mathrm{O}\right)\left(\mathrm{CH}_{3} \mathrm{CN}\right)_{2}\right]\left(\mathrm{ClO}_{4}\right)_{2}$ (2)}

The green $\left[\mathrm{Fe}\left(\mathrm{C}_{10} \mathrm{H}_{18} \mathrm{~N}_{4} \mathrm{O}\right)(\mathrm{DMF})_{2}\right]\left(\mathrm{ClO}_{4}\right)_{2}(0.490 \mathrm{~g}$ $0.80 \mathrm{mmol}$ ) was dissolved in acetonitrile, filtered and then stirred for $30 \mathrm{~min}$. A four-fold excess of diethyl ether was added to complete precipitation of the blue powdery product. Filtration followed by diethyl ether washings gave $0.400 \mathrm{~g}$ $(0.73 \mathrm{mmol})$ of product. Yield: $91 \%$.

\subsubsection{Preparation of $\left[\mathrm{Fe}\left(\mathrm{C}_{10} \mathrm{H}_{18} \mathrm{~N}_{4} \mathrm{O}\right)\left(\mathrm{CH}_{3} \mathrm{CN}_{2}\right]\left(\mathrm{PF}_{6}\right)_{2}\right.$}

An excess of $\mathrm{NH}_{4} \mathrm{PF}_{6}$ (about 5:1) was added to a slurry of the $\left[\mathrm{Fe}\left(\mathrm{C}_{10} \mathrm{H}_{18} \mathrm{~N}_{4} \mathrm{O}\right)\left(\mathrm{CH}_{3} \mathrm{CN}\right)_{2}\right]\left(\mathrm{ClO}_{4}\right)_{2}(0.400 \mathrm{~g} .0 .73$ $\mathrm{mmol})$ in acetonitrile $(25 \mathrm{ml})$. After all the colored solids dissolved, the solution was filtered. The addition of an eightfold excess of diethyl ether and cooling of the solution overnight completed the precipitation of the blue powdery product, which was obtained after filtration and diethyl ether washings. Yield: $0.38 \mathrm{~g}(0.60 \mathrm{mmol}, 82 \%)$. Anal. Calc. for $\mathrm{FeC}_{14} \mathrm{H}_{24} \mathrm{~N}_{6} \mathrm{OP}_{2} \mathrm{~F}_{12}$ : Fe, 8.75; C, 26.35; $\mathrm{H}, 3.79 ; \mathrm{N}, 13.17$; Found: Fe, 8.81; C, 26.45; H, 3.81; N, 13.10\%.

\section{Results and discussion}

We first discuss the various data supporting the assignment of the structure of 2 with particular emphasis on the proton NMR spectrum. The Mössbauer, solvatochromism and electrochemistry data are then presented, followed by speculation about mechanisms of formation of the keto complex from reaction of 1 with dioxygen.

\subsection{Structure assignment}

\subsubsection{Spectroscopy}

The optical spectrum of $\mathbf{2}$ in acetonitrile has a single band in the visible region $(650 \mathrm{~nm})$ that is too intense to be a d-d transition $\left(\epsilon=3660 \mathrm{M}^{-1} \mathrm{~cm}^{-1}\right.$ ). In reviewing the iron macrocycle literature we noted a paper by Riley and Busch [3] which described the formation of a series of diketo

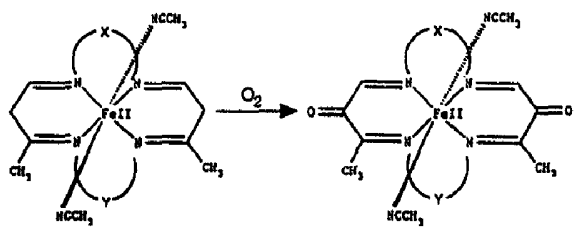

$X, Y=2,3$ 
macrocycles by reaction between dioxygen and $\boldsymbol{\beta}$-diimine macrocycles. These keto species were all blue in acetonitrile with a single visible peak at $650 \mathrm{~nm}$. The similarity in optical spectra suggested that a similar chromophore was present in compound 2. Similar reactivity between dioxygen and cobalt $\beta$-diimine macrocycles had earlier been described by Endicott and co-workers [4].

The mass spectral results support the monomeric nature of 2. The predominant observed peak in electrospray MS is at $m / z=365$ which corresponds to $\left[\mathrm{Fe}\left(\mathrm{C}_{10} \mathrm{H}_{18} \mathrm{~N}_{4} \mathrm{O}\right)\right.$ $\left.\left(\mathrm{ClO}_{4}\right)\right]^{+1}$, i.e., 2 with the loss of the axial acetonitrile ligands and inclusion of a perchlorate counter ion.

The IR spectrum of compound $\mathbf{2}$ indicates the presence of a carbonyl. The IR spectrum of the starting dinuclear compound 1 has $C=N$ peaks of moderate intensity at 1610 and $1640 \mathrm{~cm}^{-1}$. The IR spectrum of 2 has a peak at $1650 \mathrm{~cm}^{-1}$ with much greater intensity than neighboring peaks. This peak is in the $\mathbf{C}=\mathbf{O}$ spectral region and is similar to those observed by Riley and Busch [3] and Endicott and co-workers [4] in their $\beta$-diimine keto species.

\subsubsection{NMR}

The ${ }^{13} \mathrm{C}$ and ${ }^{1} \mathrm{H}$ NMR spectra of compound 2 confirm its structural assignment. The proposed structure has a plane of symmetry through the carbonyl group, the iron atom and the opposite methylene carbon atom. This plane is perpendicular to the general plane of the macrocycle; the same symmetry element is present [1] in the crystal structure of 1 . The ketomacrocycle has six different kinds of carbon atoms and this is observed in the ${ }^{13} \mathrm{C}$ NMR spectrum. The carbonyl carbon is observed at $187.7 \mathrm{ppm}$, relative to TMS, the olefinic carbon at $176.4 \mathrm{ppm}$ and the four methylene carbon resonances range from 59.7 to $33.3 \mathrm{ppm}$. It should be noted that especially long delay times of $10 \mathrm{~s}$ between pulses are necessary to detect the carbonyl carbon resonance.
Ten resonances in the ${ }^{1} \mathrm{H}$ NMR spectrum are expected if the proposed conformation is locked on the NMR time scale. The ten resonances result from the fact that the protons at the equatorial and axial positions of a given carbon are nonequivalent. The ${ }^{1} \mathrm{H}$ NMR spectrum of complex 2 in acetonitrile- $\mathrm{d}_{3}$ does indeed exhibit ten different resonances. Fig. 1 shows the ${ }^{1} \mathrm{H}$ NMR spectrum of $\mathbf{2}$ and gives the numbering scheme. A summary of the assignments and coupling constants is given in Table 1. The resonances assigned to positions $8 \mathrm{a}$ and $8 \mathrm{e}$ are the most upfield and integrate to half the area of the other positions. These resonances at approximately 2.0 and $1.75 \mathrm{ppm}$ are partially obscured by residual, undeuterated acetonitrile and by axial ligand exchange with the deuterated solvent. These upfield multiplets are not obscured when DMSO- $d_{6}$ is used as the solvent. Although the multiplet pattems are not as well resolved in DMSO- $d_{6}$, compared to $\mathrm{CD}_{3} \mathrm{CN}$, the upfield multiplets still integrate to half intensity. We have used 2-D, conventional homonuclear decoupling and a limited number of NOE difference experiments to assign the observed 'HNMR resonances to specific positions in our proposed structure.

The peak at $8.77 \mathrm{ppm}$ is assigned to the olefinic protons at position 2 of our model. The olefinic peak is slightly split due to a small four-bond coupling with the proton at position $4 \mathrm{a}$ $(4.06 \mathrm{ppm})\left(J_{4 \mathrm{a}_{2}}=2.4 \mathrm{~Hz}\right)$. Also, the short through-space distance between these two protons has been confirmed by NOE experiments. A broad signal at $4.76 \mathrm{ppm}$ is assigned to the amine protons. Amine protons typically exhibit broad NMR signals due to the quadrupole moment of the ${ }^{14} \mathrm{~N}$ nucleus. This signal disappears upon treatment with $\mathrm{DCl}$, which confirms its assignment.

The assignment of other resonances was done by determining the coupling patterns with spin-decoupling experiments and a 2D COSY experiment (supplemental). In a spin-decoupling experiment, the amine proton at $4.76 \mathrm{ppm}$ was found to be coupled strongly with the resonances at 2.50
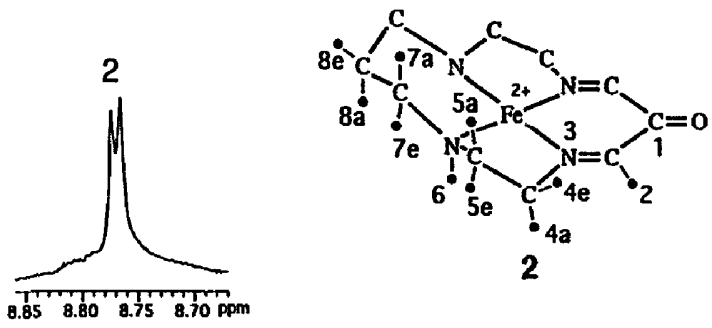

2

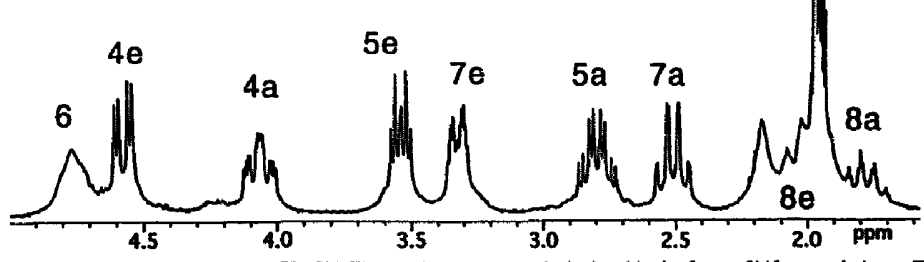

Fig. 1. Proton NMR of complex 2 in $\mathrm{CD}_{3} \mathrm{CN}$. The numbering scheme is depicted in the figure. Shifts are relative to TMS. 
Table 1

Proton assignments and coupling constants for complex $2^{\text {ab }}$

\begin{tabular}{|c|c|c|c|}
\hline $\begin{array}{l}\text { Chemical shift } \\
\text { (ppm) }\end{array}$ & Relative integral & Assignment & $\begin{array}{l}\text { Coupling constant } \\
(\mathrm{Hz})\end{array}$ \\
\hline 8.77 & 2 & $\mathrm{H} 2$ & $J_{4 \mathrm{~s} 2}(2.4)$ \\
\hline 4.76 & 2 & H 6 & broad signal \\
\hline 4.56 & 2 & $\mathrm{H} 4 \mathrm{e}$ & $J_{40 \mathrm{da}}(14.5), J_{4 \mathrm{es}, \mathrm{a}}(4.4)$ \\
\hline 4.06 & 2 & $\mathbf{H} 4 \mathbf{a}$ & $\begin{array}{l}J_{4 \mathrm{a} 4 \mathrm{c}}(14.5), J_{4 \mathrm{asa}}(14.5) \\
J_{4 \mathrm{a} .5 \mathrm{c}}(5.2), J_{4 \mathrm{a} 2}(2.4)\end{array}$ \\
\hline 3.53 & 2 & H $5 e$ & $\begin{array}{l}J_{5 \mathrm{csa}}(12.6), J_{5 \mathrm{c}+\mathrm{A}_{\mathrm{a}}}(5.2) \\
J_{5 \mathrm{co}}(4.5)\end{array}$ \\
\hline 3.31 & 2 & H 7e & $\begin{array}{l}J_{7 \mathrm{eqa}}(11.8), J_{7 \mathrm{eb}}(3.8) \\
J_{7 \mathrm{egv}}(3.8), J_{7 \mathrm{e} \mathrm{Ba}_{\mathrm{B}}}(2.4)\end{array}$ \\
\hline 2.78 & 2 & H 5a & $\begin{array}{l}J_{5 \mathrm{ab}}(12.6), J_{5 \mathrm{ase}}(12.6) \\
J_{5 \mathrm{ada}}(14.5), J_{5 \mathrm{aac}}(4.4)\end{array}$ \\
\hline 2.50 & 2 & H 7a & $\begin{array}{l}J_{7 \mathrm{ab}}(11.8), J_{\mathrm{agc}}(11.8) \\
J_{7 \mathrm{aga}}(11.8), J_{7 \mathrm{agc}}(1.7)\end{array}$ \\
\hline 2.00 & 1 & $\mathrm{H} 8 \mathrm{e}$ & obscured \\
\hline 1.75 & 1 & H $8 \mathbf{a}$ & obscured \\
\hline
\end{tabular}

a Coupling constants are given as absolute values.

"Chemical shifts are rejative to TMS.

and $2.78 \mathrm{ppm}$ and weakly to those at 3.31 and $3.53 \mathrm{ppm}$. These resonances must be due to the protons on carbons 5 and 7 . The carbon 7 protons were assigned to the peaks at 2.50 and $3.31 \mathrm{ppm}$ because they were the only ones coupled to the half-integrated intensity upfield protons of position 8 ( 2.0 and $1.75 \mathrm{ppm}$ ). The proton resonances at 4.06 and 4.56 ppm were assigned to position 4 because they were coupled to the carbon 5 protons ( 2.78 and $3.53 \mathrm{ppm}$ ). These assignments were confirmed by working back from the unique olefinic proton.

In rigid cyclic systems the equatorial proton is consistently downfield relative to the axial proton on the same carbon atom. This is the situation for complex 2; however, the differences in chemical shifts of the equatorial and axial protons are larger than the usual 0.1 to $0.5 \mathrm{ppm}$ range: $\Delta(4 \mathrm{e}-4 \mathrm{a})$ $=0.52 \mathrm{ppm} ; \Delta(5 \mathrm{e}-5 \mathrm{a})=0.74 \mathrm{ppm} ; \Delta(7 \mathrm{e}-7 \mathrm{a})=0.82$ $\mathrm{ppm}$. A consequence of this is the occurrence of the signal of the $7 \mathrm{e}$ proton between those of the $5 \mathrm{e}$ and 5 a protons in the spectrum. Individual coupling constants were determined by successive decoupling experiments and measurements of resultant peak positions. The observed multiplet patterns were also reproduced by computer simulations using the experimental coupling constants reported in Table 1 .

The optical, electrospray MS, IR and particularly the ${ }^{13} \mathrm{C}$ and ${ }^{1} \mathrm{H}$ NMR taken together allow confident assignment of the structure of complex 2 . We now consider specific aspects of the optical, Mössbauer and electrochemical data of 2.

\subsection{Physical properties}

\subsection{Optical spectra}

The absorption spectrum of complex 2 in acetonitrile (Fig. 2) exhibits maxima at $650 \mathrm{~nm}\left(\epsilon=3660 \mathrm{M}^{-1} \mathrm{~cm}^{-1}\right)$, $340 \mathrm{~nm}\left(\epsilon=2470 \mathrm{M}^{-1} \mathrm{~cm}^{-1}\right)$ with a shoulder at $370 \mathrm{~nm}$ and at $232 \mathrm{~nm}\left(\epsilon=8890 \mathrm{M}^{-1} \mathrm{~cm}^{-1}\right)$.
As mentioned earlier our initial designation of 2 as a ketomacrocycle was based on the similarity of its optical spectrum to iron keto $\beta$-diimine species previously reported by Riley and Busch [3]. These workers assigned the transition at 650 $n m$ as an MLCT from iron $d_{x z}, d_{y z}$ orbitals to a $\pi^{*}$ orbital of the ligand. We agree with this assignment for complex 2. Solutions of $\mathbf{2}$ in different solvents have very different colors - blue in acetonitrile and olive-green $\left(\lambda_{\max }=720 \mathrm{~nm}\right)$ in DMF. The change in optical spectra results from a change in axial ligands. The addition of chloride ion to an acetonitrile solution of 2 also results in a red shift ( $\lambda_{\max }=810 \mathrm{~nm}$ with $\mathrm{Cl}^{-}$) of the spectral peaks (Fig. 2). The simplest explanation is that the energies of metal $\mathrm{d} \pi$ orbitals increase as the axial ligand changes from $\mathrm{CH}_{3} \mathrm{CN}$ to $\mathrm{Cl}^{-}$. Thus the shifts to longer wavelengths result from bringing the metal orbitals closer in

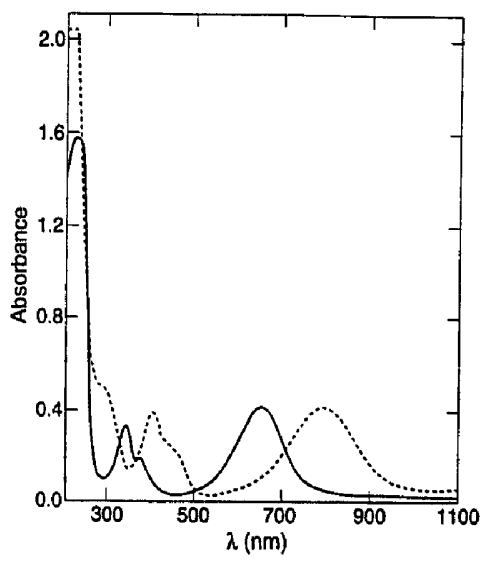

Fig. 2. Optical spectra of 2 in $\mathrm{CH}_{3} \mathrm{CN}(\ldots \ldots)$ and in $\mathrm{CH}_{3} \mathrm{CN}$ with $0.05 \mathrm{M}$ tetraethylammonium chloride $(\cdots)$. 
energy to the lowest unoccupied ligand orbitals. Since-all the bands shift, this might imply that all the transitions involve some type of charge transfer.

\subsubsection{Mössbauer spectra}

The Mössbauer spectrum of the verchlorate salt of 2 with acetonitrile axial ligands at $90 \mathrm{~K}$ consists of a quadrupole split doublet with a center shift of $0.36 \mathrm{~mm} \mathrm{~s}^{-1}$ and a quadrupole splitting of $1.62 \mathrm{~mm} \mathrm{~s}^{-1}$. The values for the binuclear compound, 1, are very similar [5]: 0.34 and $1.63 \mathrm{~mm} \mathrm{~s}^{-1}$. The Mössbauer parameters for 2 can be compared to those for a large series of 14-membered tetraaza macrocyclic lowspin Fe(II) complexes studied by Busch and co-workers [6] . The literature complexes have the same 2,3,2,3 carbon linkages between the nitrogens, have axial $\mathrm{CH}_{3} \mathrm{CN}$ ligands and have perchlorate as the counter-anion so they are directly comparable. Mössbauer parameters are available for a series of such complexes ranging from the fully saturated species to those with 2,3 and 4 carbon-nitrogen double bonds. (See table in Ref. [5].) The center shift value of $0.36 \mathrm{~mm} \mathrm{~s}^{-1}$ is at the low range for this extensive series of closely-related complexes while the quadrupole splitting value is high. The center shift parameter is inversely related to the s electron density at the iron nucleus [7]. Good sigma-donor and good pi-acceptor ligands both lead to increased $s$ density and hence to lower center shift values. While $\sigma$ - and $\pi$-bonding properties of ligands are additive in determining the center shift value, the quadrupole splitting is a measure of the difference between these two properties.

Busch and co-workers [6] concluded that $\pi$ effects predominate in tetraaza marrocycles with carbon-nitrogen double bonds because they found a negative slope in a plot of quadrupole splitting versus center shift for their series. The low center shift and high quadrupole splitting values for 2 clearly indicate that the keto-macrocyclic ligand is an excellent $\pi$-acceptor. The carbonyl oxygen evidently attracts electron density and greatly increases the $\pi$-acid capabilities of the imine nitrogens. Only the cross-conjugated binucleating ligand 1 has similar Mössbauer parameters and this binucleating ligand has also bcen identified [5] as an excellent $\pi$ acceptor ligand.

\subsubsection{Electrochemistry}

The $\mathrm{CV}$ of complex 2 in $\mathrm{CH}_{3} \mathrm{CN}$ shows an irreversible oxidation at $930 \mathrm{mV}$ versus $\mathrm{Fc} / \mathrm{Fc}^{+}$(Fig. 3(a)). This is a very positive potential for oxidation of $\mathrm{Fe}^{2+}$ to $\mathrm{Fe}^{3+}$. The stabilization of $\mathrm{Fe}^{2+}$ by the keto $\beta$-diimine ligand is due to its exceptional $\pi$-acceptor properties and the greater $\pi$-donor strength of iron in the +2 oxidation state relative to the +3 level. There is a reversible one-electron reduction in $\mathrm{CH}_{3} \mathrm{CN}$ at $-980 \mathrm{mV}$. This reduction may correspond to putting an electron in the keto $\beta$-diimine moiety of the ligand. There is also a second broad, poorly defined reduction wave at -1360 $\mathrm{mV}$. The broad and irreversible nature of this wave is not well understood but may be due to a process corresponding

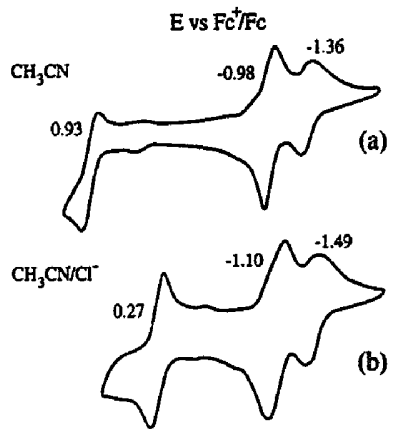

DMF

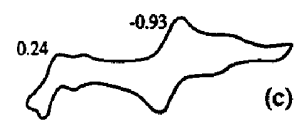

Fig. 3. Cyclic voltammograms of complex 2 in $\mathrm{CH}_{3} \mathrm{CN}, \mathrm{CH}_{3} \mathrm{CN}$ with $0.1 \mathrm{M}$ tetraethylammonium chloride and in DMF. Supporting electrolyte was $0.1 \mathrm{M}$ tetrabutylammonium hexafluorophosphate in $\mathrm{CH}_{3} \mathrm{CN}$ and DMF but no additional electrolyte was added to the $\mathrm{CH}_{3} \mathrm{CN} / \mathrm{Cl}^{-}$solution. Conditions: scan rate $200 \mathrm{mV} \mathrm{s}^{-1}$, platinum working and counter electrodes, potentials measured vs. ferrocinium/ferrocene couple. A small amount of impsrity of the original binuclear species 1 is observed at potentials just negative of the $\mathrm{Fe}^{\mathrm{II}} / \mathrm{Fe}^{\mathrm{III}}$ oxidation.

to pinacol reduction with the protons coming from adventitious water.

The optical spectrum of 2 with $\mathrm{CH}_{3} \mathrm{CN}$ shows a pronounced red shift when chloride ion is added or when 2 is dissoived in DMF. The visible band is assigned as an MLCT and the spectral shifts are assumed to be due to changes in the energies of the metal orbitals as $\mathrm{Cl}^{-}$or DMF replace $\mathrm{CH}_{3} \mathrm{CN}$ as the axial ligands. If these assumptions are true, the electrochemical behavior should also exhibit an axial ligand dependence.

The electrochemistry does indeed show a marked shift with a $600 \mathrm{mV}$ lower oxidation potential when $\mathrm{Cl}^{-}$is added (Fig. 3(b)). Also, the oxidation wave with added $\mathrm{Cl}^{-}$is more reversible but this may just reflect the lower oxidation potential and hence greater stability of iron in the +3 oxidation state with $\mathrm{Cl}^{-}$axial ligands.

There is also an electrostatic factor to be considered. The keto $\mathrm{Fe}$ (II) macrocyclic species has a +2 charge when the axial ligands are $\mathrm{CH}_{3} \mathrm{CN}$ but is neutral with $\mathrm{Cl}^{-}$axial ligands. Everything else being equal, it will be easier to remove an electron from a neutral species than from one with $\mathbf{a}+2$ overall charge. However, there is a similar large shift ( 690 $\mathrm{mV}$ ) to a less positive oxidation potential when the axial ligands are DMF (Fig. 3(c)). The complexes with DMF and with $\mathrm{CH}_{3} \mathrm{CN}$ axial iigands both have a +2 overall charge so we infer that the electrostatic effect is not the main reason for the shift in oxidation potential when $\mathrm{Cl}^{-}$replaces $\mathrm{CH}_{3} \mathrm{CN}$ as the axial ligands. There remains some ambiguity in comparison of oxidation potentials of species with DMF and with $\mathrm{CH}_{3} \mathrm{CN}$ axial ligands due to the change in solvent. The 
ferrocinium/ferrocene reference scale minimizes such solvent effects.

\subsection{Mechanism of formation}

The reaction of molecular oxygen with the central crossconjugated carbon-carbon bond in the binuclear species is unusual and is not affected by the absence of light. We are not aware of any examples in the organic literature that involve non-photolytic atmospheric pressure, room temperature attack of dioxygen on conjugated olefins with ketones as the only product. With crystalline salts of $1\left(\mathrm{CH}_{3} \mathrm{CN}\right.$ axial ligands) a surface film of the blue species forms over periods of weeks. If the solid is finely divided, the reaction with atmospheric oxygen proceeds more rapidly, with up to $15 \%$ conversion to 2 in 1 day.

\subsubsection{Axial ligand effects}

Oxygen can react directiy with the solid and the reaction also occurs in solution. Interestingly, the rate of this reaction is strongly solvent dependent. In oxygen-saturated acetonitrile solutions, the reaction has a half-life of over a week. In oxygen-saturated DMF the half-life for formation of $\mathbf{2}$ is less than a minute. This is greater than a factor of $10^{4}$ increase in reaction rate. One possible explanation for the very remarkable difference in the rates in DMF and $\mathrm{CH}_{3} \mathrm{CN}$ involves formation of a mixed-valent $(\mathrm{Fe}, \mathrm{Fe})^{5+}$ intermediate since $\mathrm{O}_{2}$ has the potential to oxidize 1 in DMF but not in $\mathrm{CH}_{3} \mathrm{CN}$. However, several isosbestic points are observed in sequential spectra in DMF during the reaction between 1 and dioxygen (Fig. 4). The mixed-valent compound with DMF axial ligands has a $\lambda_{\max }$ at $940 \mathrm{~nm}$ with a large molar absorbancy coefficient ( $>15000 \mathrm{M}^{-1} \mathrm{~cm}^{-1}$ ) [8]. Therefore, it would not take a large percentage build-up of the mixed-valent form to disrupt the low intensity isosbestic point at $900 \mathrm{~nm}$

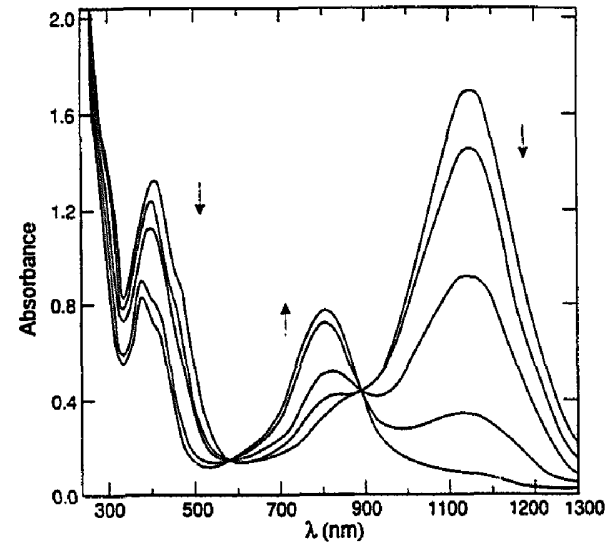

Fig. 4. Sequential spectra taken during the reaction of dioxygen with binuclear species 1 in DMF. Conditions: $5 \times 10^{-4} \mathrm{M}$ complex 1 , room temperature, open cuvette with stirring, total time $20 \mathrm{~min}$.
(Fig. 4). Experiments were done in which small aliquots with about one-fourth an equivalent of dioxygen were sequentially introduced into a septum-capped cuvette containing a solution of 1 in DMF. The isosbestic points were maintained, implying that if a mixed-valent intermediate is formed in DMF it must react extremely rapidly. However, when the mixed-valent species is produced by electrochemical oxidation of 1 in anaerobic DMF and then air is introduced, a relatively slow (minutes) reaciion occurs to give partial return to the origina? isovalent compound. This process may involve disproportionati a or reaction with water since there is no evidence of formation of 2.

Several lines of indirect evidence suggest that solvent effects are not major factors in the differences in rates in $\mathrm{CH}_{3} \mathrm{CN}$ and DMF. The reaction to form 2 was investigated in a mixed solvent with mole fraction $\mathrm{CH}_{3} \mathrm{CN} 0.9$, DMF 0.1 . Total conversion to 2 in this dioxygen-saturated $\mathrm{CH}_{3} \mathrm{CN}$-rich solvent was accomplished in $1 \mathrm{~h}$ compared to weeks in pure $\mathrm{CH}_{3} \mathrm{CN}$. The electrochemical behavior and optical spectrum in this mixed solvent were very similar to those in pure $\mathrm{CH}_{3} \mathrm{CN}$ suggesting that $\mathrm{CH}_{3} \mathrm{CN}$ molecules were the predominant axial ligands. Our interpretation is that in the mixed solvent the reaction to form 2 proceeds by reaction of dioxygen with the small equilibrium percentage of binuclear species with DMF axial ligands.

$$
\begin{array}{rl}
{\left[\mathrm{Fe}_{2} \mathrm{~B}\left(\mathrm{CH}_{3} \mathrm{CN}\right)_{4}\right]^{4+}} & +n \mathrm{DMF} \\
& \\
& \neq\left[\mathrm{Fe}_{2} \mathrm{~B}\left(\mathrm{CH}_{3} \mathrm{CN}\right)_{4-n}(\mathrm{DMF})_{n}\right]^{4+} \\
& \downarrow 0_{2} \\
2 & 2
\end{array}
$$

$\mathrm{B}=\mathrm{C}_{20} \mathrm{H}_{36} \mathrm{~N}_{8}, \quad n=1-4$

The data do not address the interesting question of the number of DMF molecules required to activate 1 for attack by dioxygen.

Another observation points to the importance of the properties of the axial ligands and absence of major contributions by solvent effects in determining rates and paths of reactions. Addition of tetraethylammonium chloride to a DMF or $\mathrm{CH}_{3} \mathrm{CN}$ solution of 1 produces a yellow chloro species [8], $\left[\mathrm{Fe}_{2}\left(\mathrm{C}_{20} \mathrm{H}_{36} \mathrm{~N}_{8}\right) \mathrm{Cl}_{4}\right]^{0}$. When $\mathrm{O}_{2}$ is then introduced to either a $\mathrm{CH}_{3} \mathrm{CN}$ or DMF solution of the isovalent chloro species, a red mixed-valent ion $\left[\mathrm{Fe}_{2}\left(\mathrm{C}_{20} \mathrm{H}_{36} \mathrm{~N}_{8}\right) \mathrm{Cl}_{4}\right]^{+1}$ is rapidly formed in good yield ( $>95 \%)$. Clearly the solvent has little effect and the product of the reaction is determined by the chloride axial ligands.

\subsubsection{Proposed mechanism}

The high yield of ketone and the observation that the reaction also occurs in the solid state suggests that a dioxetane intermediate is involved in the mechanism. Dioxetane species are known to produce high yields of ketones and a concerted cleavage to form two keto-macrocycle monorners can occur with a minimum of atomic displacement and therefore is feasible in the solid state. 


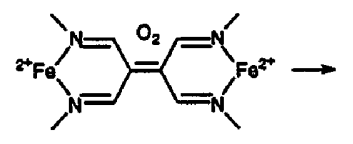

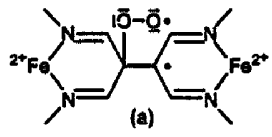

(a)
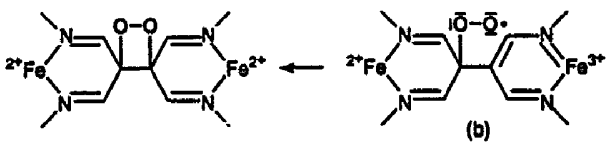

(b)

Scheme 1.

At room temperature, the reaction between the dinuclear iron compound and dioxygen in DMF occurs with no appreciable build-up of intermediates as evidenced by several isosbestic points during the reaction (Fig. 4). The reaction between 1 and dioxygen in DMF was also run at low temperature. These experiments at $-45^{\circ} \mathrm{C}$ were not successful in either isolating or in detecting intermediates via optical spectroscopy. Nevertheless, a mechanism involving reactive dioxetane intermediate seems very reasonable.

There are many examples of dioxetane intermediates formed from concerted reactions of oxygen with olefins in organic systems [9], but these require singlet oxygen, usually produced photochemically. The formation of a hydroperoxide species ( $\mathrm{ROOH}$ ) that subsequently yields the dioxetane intermediate would remove the necessity of invoking singlet oxygen. However, formation of a hydroperoxide intermediate requires the presence of an allylic hydrogen atom which is absent in the cross-conjugated ligand in $\mathbf{1}$.

From the paucity of similar examples from the organic literature of facile non-photolytic room temperature atmo:pheric-pressure attack of dioxygen on conjugated olefins, it is likely that the iron centers in $\mathbf{1}$ are directly involved. Also, the very large effect of the axial ligands of the iron on the rate of the reaction must be taken into account. A plausible stepwise mechanism for dioxetane formation is shown in Scheme 1 . The first step proceeds via transfer of one electron from the $\pi$-system to a dioxygen molecule. This produces a peroxide radical with concomitant formation of another radical in a six-membered chelate ring (species a). The total spin can be conserved in this step. A resonance form of species a has a quasi-aromatic arrangement with oxidation of low-spin iron II to low-spin iron III. The necessary next step involves triplet-to-singlet intersystem conversion followed by combination of the intramolecular radical pair to form the dioxetane intermediate. Any change on the iron center which permits easier oxidation such as replacing $\mathrm{CH}_{3} \mathrm{CN}$ with DMF axial ligands should lower the aciivation energy and accel- erate the reaction. Another way of describing the situation is that an increase in the $\pi$-donor ability of iron will stabilize the transition state. Axial ligands with $\pi$-acid properties such as $\mathrm{CH}_{3} \mathrm{CN}$ may compete with the bridging ligand for iron $\mathrm{d} \pi$ electrons whereas sigma donor ligands such as DMF may increase the $\pi$-donor ability of iron. A reasonable assumption is that the iron centers are able to facilitate triplet-to-singlet conversion and this is the reason why there are so few examples of similar reactivity in pure organic systems.

When chlorides are the axial ligands, dioxygen removes a single electron from the binuclear compound 1 to produce a mixed-valent species. In the electrochemical experiments the $E^{0}$ s for oxidation of 1 with DMF and with $\mathrm{Cl}^{-}$axial ligands are similar. There are stringent stereo and electronic requirements for formation of the four-center dioxetane species and relatively subtle modifications could result in the very different products of the reaction of dioxygen with complex 1 with different axial ligands.

We are continuing our studies of the chemistry of the keto $\boldsymbol{\beta}$-diimine iron complexes and are focusing on using the keto functional group as a coupling agent to produce other interesting systems.

\section{Acknowledgements}

Acknowledgement is made to the donors of the Petroleum Research Fund, administered by the American Chemical Society for partial support of this research. This work was also supported in part by the Diretor, Office of Energy Research, Office of Basic Energy Sciences, Chemical Sciences Division, of the U.S. Department of Energy under Contract No. DE-AC03-767FS00098.

\section{References}

[1] H.S. Mountford, L.O. Spreer, J. Otvos, M. Calvin, KJ. Brewer, M. Richter and B. Scoth, Inorg. Chem., 31 (1992) 718.

[2] J. Zhao, A. Li, C.B. Allaa, L.O. Spreer, J.W. Otvos and M. Calvia, Inorg. Chim. Acta., 246 (1996) 241.

[3] D.P. Riley and D.H. Busch, Inorg. Chem., 22 (1983) 4141.

[4] B. Durham, T.J. Anderson, J.A. Switzer, J.F. Endicott and M.D. Glock, Inorg. Chem., 16 (1977) 271.

[5] H.S. Mountford, D.B. MacQueen, A. Li, J.W. Otros, M. Calvin, R.B. Frankel and L.O. Spreer, Inorg. Chem., 33 (1994) 1748.

[6] J.C. Dabrowiak, P.H. Merrell, J.A. Stone and D.H. Busch, J. Am. Chem. Soc., 95 (1973) 6613.

[7] R.S. Drago, Physical Methods for Chemisis, Saunders, Philadelphia, PA. 2nd edn., 1992, pp. 5430-5448.

[8] A. Li, M.SC. Thesis, University of the Pacific. Stockton, CA. USA, 1993.

[9] A.A. Frimer, Chem. Rev., 79 (1979) 359. 